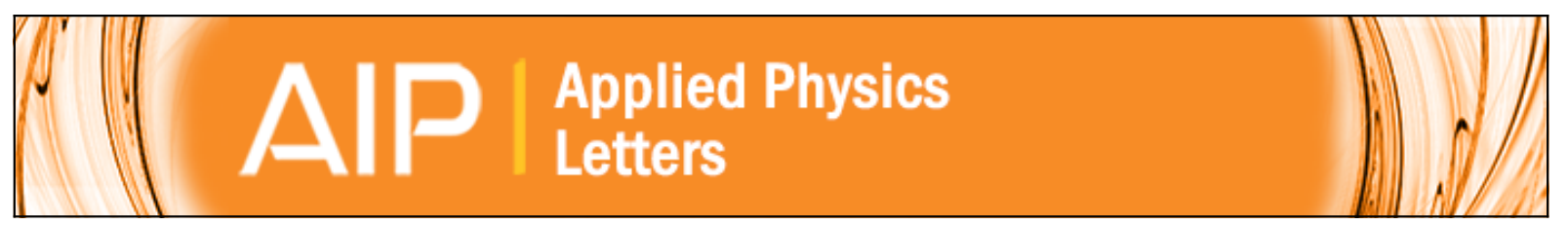

\title{
Microscopic origins of dry-etching damages in silicon large-scaled integrated circuits revealed by electrically detected magnetic resonance
}

K. Uejima and T. Umeda

Citation: Applied Physics Letters 104, 082111 (2014); doi: 10.1063/1.4867070

View online: http://dx.doi.org/10.1063/1.4867070

View Table of Contents: http://scitation.aip.org/content/aip/journal/apl/104/8?ver=pdfcov

Published by the AIP Publishing

\section{Articles you may be interested in}

Generating integrated-circuit patterns via cutting and stitching of gratings

J. Vac. Sci. Technol. B 27, 2750 (2009); 10.1116/1.3264677

Backside observation of large-scale integrated circuits with multilayered interconnections using laser terahertz emission microscope

Appl. Phys. Lett. 94, 191104 (2009); 10.1063/1.3133346

Monolithically integrated low-loss silicon photonic wires and three-dimensional tapered couplers fabricated by self-profile transformation

Appl. Phys. Lett. 91, 191114 (2007); 10.1063/1.2809359

Electrically detected magnetic resonance of ion-implantation damage centers in silicon large-scale integrated circuits

J. Appl. Phys. 94, 7105 (2003); 10.1063/1.1623608

End-point detection using focused ion beam-excited photoemissions in milling deep small holes in large scale integrated circuit structures

J. Vac. Sci. Technol. B 16, 2555 (1998); 10.1116/1.590209

\section{NEW! Asylum Research MFP-3D Infinity ${ }^{\text {TM }}$ AFM}

Unmatched Performance, Versatility and Support

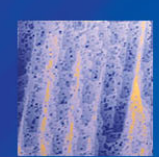

Comprehensive tools for nanomechanics
Stunning high performance
Simpler than ever to GetStarted ${ }^{\mathrm{TM}}$

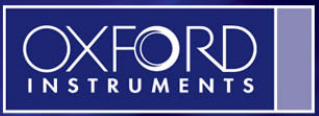

The Business of Science ${ }^{\mathrm{e}}$

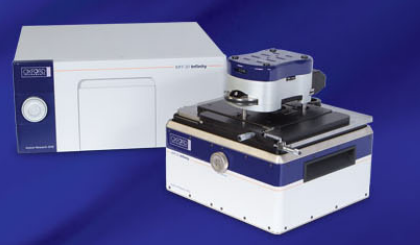




\title{
Microscopic origins of dry-etching damages in silicon large-scaled integrated circuits revealed by electrically detected magnetic resonance
}

\author{
K. Uejima ${ }^{1,2, a)}$ and T. Umeda ${ }^{1}$ \\ ${ }^{1}$ Institute of Applied Physics, University of Tsukuba, Tsukuba 305-8573, Japan \\ ${ }^{2}$ Renesas Electronics Corporation, Sagamihara 252-5298, Japan
}

(Received 6 December 2013; accepted 11 February 2014; published online 27 February 2014)

\begin{abstract}
We reveal microscopic structures of dry-etching damage defects in practical Si metal-oxidesemiconductor field effect transistors. Electrically detected magnetic resonance spectroscopy identified interstitial defects of carbon (a split C-Si interstitialcy) and fluorine (a bond-centered fluorine) as the major dry-etching damages, which survived even through high-temperature thermal processes. In addition, we found other minor centers of carbon, fluorine, and possibly hydrogen impurities. Our observation indicates that the observed defects became much more stable than those in bulk silicon. (C) 2014 AIP Publishing LLC. [http://dx.doi.org/10.1063/1.4867070]
\end{abstract}

Carbon and fluorine are the major impurities in silicon large-scale integration circuits (Si LSIs). They are intentionally introduced by co-implantation in order to form an abrupt junction profile of Si metal-oxide-semiconductor field effect transistors (MOSFETs). ${ }^{1,2}$ In the implanted regions, carbon and fluorine atoms can trap excess interstitial Si atoms and strongly suppress the transient enhanced diffusion of boron and phosphorous dopants. ${ }^{1,2}$ They are also unintentionally introduced by dry etching. ${ }^{3,4}$ Fluorocarbon containing fluorine and carbon is one of the most popular gases for reactive ion etching (RIE) of $\mathrm{SiO}_{2}$ and $\mathrm{SiN}$ films. The energetic flux of carbon and fluorine damages the crystalline Si lattice and eventually causes the mixing of fluorine and carbon atoms with the lattice. ${ }^{4}$ Controlling of such damage caused by RIE is one of the major challenges for advanced scaled LSIs. Both carbon and fluorine atoms can create lattice defects ${ }^{5,6}$ and may become the source of the leakage currents of $p-n$ junctions. ${ }^{2,7}$ However, microscopic entities of such defects are still unclear. Since Si MOSFETs are fabricated via a number of LSI processes, the formation and survival of defects inside MOSFETs are very complicated issues.

In this Letter, we reveal the microscopic structures of carbon- and fluorine-related defects remaining in practical $\mathrm{Si}$ MOSFETs by means of electrically detected magnetic resonance (EDMR) spectroscopy. ${ }^{8}$ EDMR achieved electrical detections of electron-spin-resonance (ESR) signals in the practical devices. These defects were introduced via dryetching processes and survived even through hightemperature thermal processes. They could be reduced by shortening the etching time. This reduction of defects has also the benefit of a low junction leakage current of MOSFETs.

Figure 1 shows the depth profiles of carbon and fluorine atoms in Si substrates after an RIE process, which were measured by secondary ion mass spectrometry (SIMS). We compared the two processes. "Process A" included an etch-back of a $\mathrm{SiO}_{2}$ cover layer on $\mathrm{Si}$ substrate done by using a fluorocarbon RIE process. The etch-back was performed for a period 1.5-times longer than the etching time of just removing the $\mathrm{SiO}_{2}$ film. Therefore, the $\mathrm{Si}$ substrate was exposed to

${ }^{a)}$ E-mail: kazuya.uejima.kf@renesas.com fluorocarbon plasma. This kind of condition was conventionally used because it ensures a complete removal of the etching layer. To compare with "process A," the other substrate was subjected to "process B," in which we used a reduced RIE time 9 less than half of the period used in "process A." Figure 1 demonstrates a drastic reduction in the incorporation of carbon and fluorine atoms when we used "process B."

Following the SIMS analysis, we carried out EDMR measurements on Si MOSFETs (samples A and B) subjected to either "process A" or "process B." The device structure is schematically shown in Fig. 2(a). Arrays of $n$-channel $\mathrm{Si}$ MOSFETs (sub-100-nm gate length) were fabricated on 300mm-diameter Si(100) wafers. The boron concentration of the substrate was $\sim 1 \times 10^{18}$ atoms $/ \mathrm{cm}^{3}$. To pattern MOSFET structures, the chemical vapor deposition (CVD) of a $\mathrm{SiO}_{2}$ cover film as well as the RIE process for etching back the cover film were performed. After that, arsenic and phosphorous atoms were highly doped over $1 \times 10^{19}$ atoms $/ \mathrm{cm}^{3}$ in the source and drain regions by ion implantation. These dopants were activated by rapid thermal annealing at over $1000^{\circ} \mathrm{C}$. This led to a thermal diffusion of the dopants, and the junction depth extended to about $100 \mathrm{~nm}$. The carbon and fluorine impurities were also thermally diffused not only vertically but also laterally toward the gate area, as is drawn in Fig. 2(a).

Figure 2(b) shows the EDMR spectra at $300 \mathrm{~K}$ of samples $\mathrm{A}$ and $\mathrm{B}$ for the same junction leakage currents $(\sim 8 \mathrm{nA})$. A strong signal was observed at $333.5 \mathrm{mT}$ for sample A. We named this as "Ci." This signal was also found in sample B, but it was so weak that we needed a longer accumulation time. We checked the EDMR signal intensity (current change due to ESR) as a function of the junction voltage and found that the signal intensity was constant at $\sim 200 \mathrm{ppm}$ for sample A when the voltage was under $1.75 \mathrm{~V}$ [see the inset of Fig. 2(b)]. In comparison, it decreased to $\sim 50 \mathrm{ppm}$ for sample B. This result indicates that there should be more defects in sample A than in sample B. In fact, the junction leakage currents at $1.75 \mathrm{~V}$ were also three times higher in sample A (1.2 nA) than in sample B (380 pA). This EDMR signal was not observable under forward-biased conditions. Consequently, the observed EDMR signal is clearly related to the source of the junction leakage currents. 


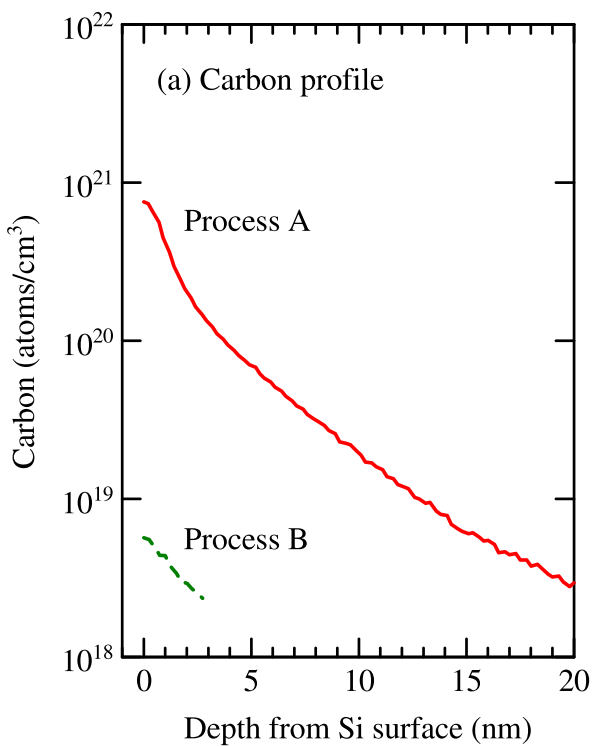

Figure 3(a) shows the details of the EDMR spectra in sample $\mathrm{A}$ and their angular dependence with respect to the magnetic-field rotation in the $(0 \overline{1} 1)$ plane. In this rotation, the major crystal directions, [100], [111], and [011], correspond to $0^{\circ}, 54.8^{\circ}$, and $90^{\circ}$, respectively. We found two major EDMR signals in Fig. 3(a). One is a stronger central signal, "Ci," which also appeared in Fig. 2(b). The second signal is a doubly split signal, which we named "Fi." We propose that "Ci" and "Fi" arise from interstitial defects of carbon and fluorine, respectively. These atoms are the most dominant impurities, as evidenced in Fig. 1. The current model naturally accounts for the drastic reduction of the two signals in sample B where the incorporation of carbon and fluorine was greatly suppressed. Furthermore, the clear doublet hyperfine splitting of " $\mathrm{Fi}$ " is consistent with that of ${ }^{19} \mathrm{~F}$ nuclear spin (natural abundance $=100 \%$, nuclear spin $I=1 / 2$ ). In comparison, there was no obvious hyperfine splitting for "Ci," in spite of its strong signal intensity. This supports the fact that " $\mathrm{C} i$ " is a carbon-related defect because carbon includes only a very small fraction of ${ }^{13} \mathrm{C}$ isotope (natural abundance $=1.1 \%, I=1 / 2$ ).

The identifications of the origins of "Ci" and "Fi" as interstitial defects are based on their angular dependences. In Fig. 3(a), we precisely fitted the EDMR spectra as displayed by the smooth overlapped lines from which we determined signal positions of "Ci" and "Fi" as well as of other weak signals. Finally, angular maps of all the observed EDMR centers are summarized in Figs. 3(b) and 3(c). Solid lines in the figures represent simulated angular maps of each center, which were calculated by using the ESR parameters in Table I. In the following sections, we will examine each defect in detail.

For main defect " $\mathrm{Ci}$," we found that its angular map is similar to that of the famous G12 center. ${ }^{10}$ Therefore, we judge that "Ci" is a close variation of G12. The G12 center has been identified as an isolated carbon interstitial in $\mathrm{Si}$, and it has an atomic structure like shown in Fig. 4. The original G12 center was found in carbon-doped highly resistive Si substrates after electron irradiation, and ${ }^{13} \mathrm{C}$-enriching experiments clearly revealed its $s p^{2}$-bonding structure and an ideal $\pi$-orbital of a $\mathrm{C} \equiv \mathrm{Si}_{3}$ unit (see Fig. 4). ${ }^{10}$ Its charge state is +1 , namely, G12 is a $\mathrm{C}_{i}(+)$ center. ${ }^{10}$ According to theoretical calculations, ${ }^{5}$ the split $\mathrm{C}$-Si interstitialcy shown in Fig. 4 is a ground-state configuration for an isolated carbon atom in $\mathrm{Si}$. Even though a carbon atom can predominantly occupy substitutional sites, this form can capture a mobile Si self-interstitial $\left(\mathrm{Si}_{i}\right)$ due to own strain field, resulting in the formation of split C-Si interstitials. 5 (a) EDMR sample and setup

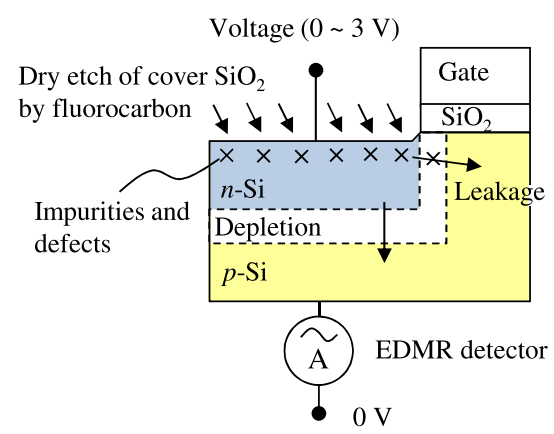

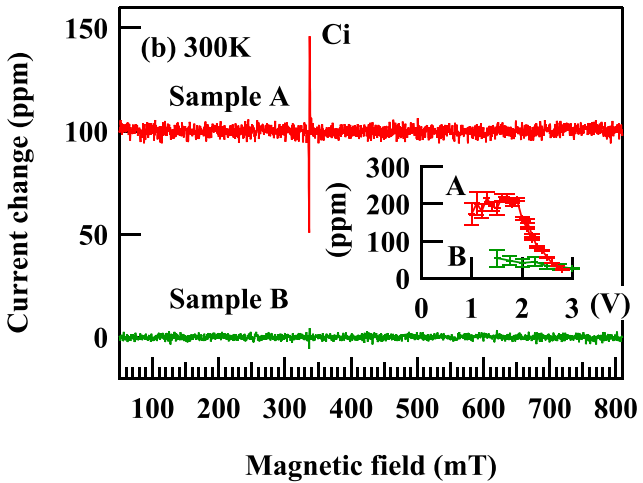

Magnetic field (mT)

FIG. 2. (a) Sample structure and EDMR setup. We activated junction currents between the $n$-type drain (or source) region and $p$-type substrate with an open gate and monitored current changes due to ESR by using an EDMR detector synchronized to a magnetic-field modulation at $1.5 \mathrm{kHz}$. (b) Typical EDMR spectra of two samples prepared by the dry-etching processes of "process A" and "process B" shown in Fig. 1. The spectra were measured by using junction leakage currents of a $\sim 8$-nA, 200 -mW microwave at $9.46 \mathrm{GHz}$, and 0.5 -mT-width magnetic-field modulation. The inset shows bias dependences of the current changes (peak-to-peak) which were measured for the junction leakage currents from $50 \mathrm{pA}$ to $75 \mathrm{nA}$. 
(a)

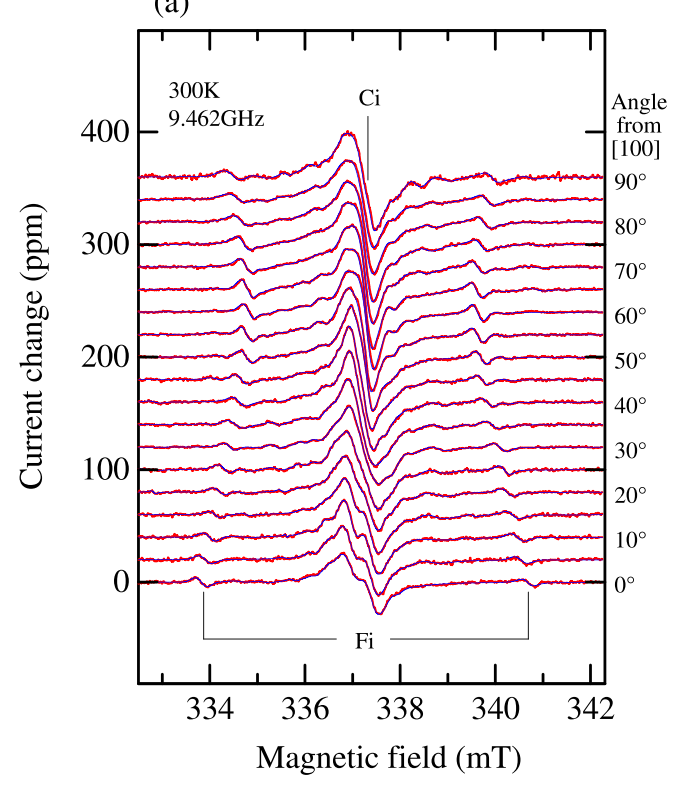

(b)

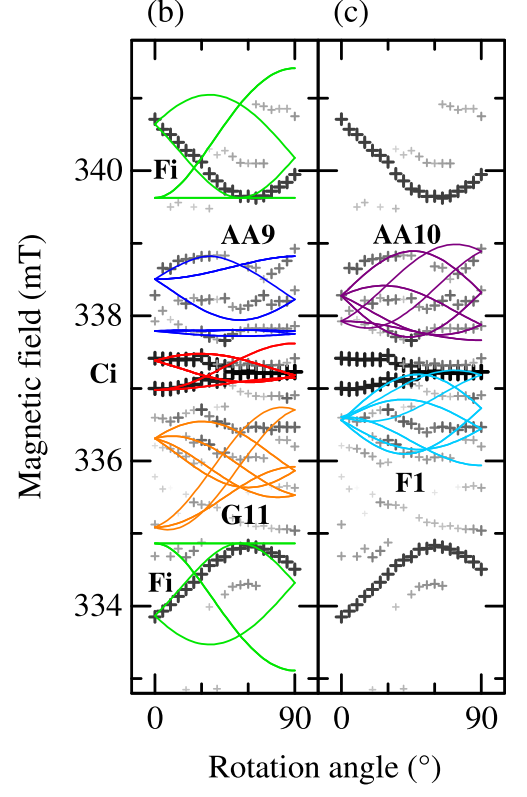

FIG. 3. (a) Angular dependence of EDMR spectra measured for a magnetic-field rotation in the $(0 \overline{1} 1)$ plane from the [100] direction $\left(0^{\circ}\right)$ to the [011] direction $\left(90^{\circ}\right)$. The spectra were measured for "sample A" by using the junction leakage currents of a $\sim 12$-nA, 200 -mW microwave at $9.46 \mathrm{GHz}$, and 0.25 -mT-width magnetic-field modulation. Two signals, labeled "Ci" and "Fi," are clearly found, in addition to many other weak signals. Smooth lines overlapping the experimental spectra represent the results of our spectral simulations, where we used the Gauss, Lorentz, and Vogit functions for each signal. Typical full widths at half maximum (FWHMs) were $0.4 \mathrm{mT}$ for "Ci" and $0.2 \mathrm{mT}$ for "Fi." (b) and (c) Experimental ("+" symbols) and simulated angular maps (solid lines) of the observed EDMR centers. Darker and larger "+" symbols represent larger experimental peaks. The "Fi" peaks are drawn more darkly for clarity. The simulations were performed by using the ESR parameters of "Ci" and "Fi" in Table I and those of G11, ${ }^{11}$ F1, ${ }^{13}$ AA9, and AA10. ${ }^{17}$

Similarly to $\mathrm{Si}_{i}, \mathrm{C}_{i}$ is also mobile, which is evident from the wider extension of SIMS profiles for carbon (Fig. 1). In the previous ESR study on irradiated bulk $\mathrm{Si}^{10}{ }^{10}$ the original G12 center was easily reduced by a low-temperature anneal at $60^{\circ} \mathrm{C}$ and was converted into the G11 center [positively charged carbon interstitial-substitutional carbon pair, $\left.\mathrm{C}_{i} \mathrm{C}_{s}(+)\right] .{ }^{10,11}$ The G11 center is formed by combining two mobile carbon atoms. The G11 center was supposedly observed in our devices because an angular-map simulation of G11 seems to fit with a part of the experimental data in Fig. 3(b). However, the signal intensity of G11 was much smaller than that of "Ci" (G12-type), despite the fact that our devices went through the high-temperature thermal processes (over- $1000^{\circ} \mathrm{C}$ activation anneal for seconds as well as over- $400{ }^{\circ} \mathrm{C}$ anneal for hours). We suggest that a large amount of excess $\mathrm{Si}_{i}$ in MOSFETs ${ }^{1,2}$ causes the dominant formation of the G12-type centers $\left[\mathrm{C}+\mathrm{Si}_{i}\right.$ rather than the $\mathrm{G} 11$ centers $\left(\mathrm{C}_{i}+\mathrm{C}_{s}\right)$. Also strongly strained $\mathrm{Si}$ lattice by the surrounding structures ${ }^{12}$ may affect the thermal stability of the defects. ${ }^{8}$ In fact, our "Ci" center exhibited slightly different ESR parameters from the original
G12 center (Table I). Therefore, we distinguish "Ci" from the original G12 center in this paper.

The "Fi" center is the secondary major defect in our devices. Its noticeable hyperfine splitting is clearly anisotropic (Fig. 3) and is in a similar range $(4.8-8.2 \mathrm{mT})$ to the case of ${ }^{19} \mathrm{~F}$ hyperfine splittings (4.2-6.3 mT) of the $\mathrm{F} 0$ center ( $\left(\mathrm{FV}_{2}\right.$ defect). ${ }^{13}$ The F0 center is a primitive type of fluorine-vacancy clusters $\left(\mathrm{F}_{n} V_{m}\right.$ defects) and is only a defect that revealed the detectable ${ }^{19} \mathrm{~F}$ hyperfine splitting in Si. However, the ${ }^{19} \mathrm{~F}$ hyperfine splittings of "Fi" and F0 exhibited such different angular dependences that we should consider opposite types of atomic structures for each center. We assign "Fi" to a bond-centered fluorine (Fig. 4) on the basis of the following reasons:

We first consider a crucial difference in their symmetries. In the F0 center, a fluorine atom forms a Si-F bond inside a $V_{2}$ space. ${ }^{6,13}$ Since this bond is nearly along the original $\mathrm{Si}-\mathrm{Si}$ bond, the ${ }^{19} \mathrm{~F}$ hyperfine splitting of F0 showed approximately a $\langle 111\rangle$ axial symmetry. ${ }^{13}$ Consequently, the hyperfine splitting was maximized in the [111] direction $\left(\sim 55^{\circ}\right)$ and was minimized in the normal direction to $[111]\left(90^{\circ}-55^{\circ}=35^{\circ}\right) .{ }^{13}$ On

TABLE I. ESR parameters of "Ci" and "Fi" EDMR centers and G12 ESR centers. The spin Hamiltonian of these centers is given by $H=\mu_{\mathrm{B}} \mathbf{S} \cdot \mathbf{g} \cdot \mathbf{B}+\mathbf{S} \cdot \mathbf{A} \cdot \mathbf{I}-$ $g_{\mathrm{n}} \beta_{\mathrm{n}} \mathbf{I} \cdot \mathbf{B},{ }^{10,13}$ where $\mathbf{g}$ is a $\mathrm{g}$ (gyromagnetic) tensor of an electron spin and $\mathbf{A}$ is a hyperfine tensor (in $\mathrm{mT}$ ) for each nuclear spin. The $\mathrm{g}$ tensor is defined by three principal $\mathrm{g}$ factors and three principal axes $(X, Y$, and $Z)$ of the tensor. The hyperfine tensor is also expressed in the same manner.

\begin{tabular}{|c|c|c|c|c|c|c|c|c|c|}
\hline \multirow[b]{2}{*}{ Center } & \multirow[b]{2}{*}{ Tensor } & \multicolumn{3}{|c|}{ Principal values } & \multicolumn{3}{|c|}{ Principal $Z$ axis } & \multirow[b]{2}{*}{ Origin } & \multirow[b]{2}{*}{ Ref. } \\
\hline & & $X$ & $Y$ & $Z(/ /)$ & $\theta$ & $\varphi$ & Axis & & \\
\hline $\mathrm{Ci}$ & g & 2.0062 & 2.0052 & 2.0024 & $90^{\circ}$ & $90^{\circ}$ & [011] & A variation of G12 & Present \\
\hline \multirow[t]{2}{*}{ G12 } & g & 2.0068 & 2.0062 & 2.0020 & $90^{\circ}$ & $90^{\circ}$ & [011] & C-Si split interstitial, $\mathrm{C}_{i}(+)$ & Ref. 10 \\
\hline & $\mathbf{A}\left({ }^{13} \mathrm{C}\right)$ & 0.67 & 0.63 & 5.20 & $0^{\circ}$ & $0^{\circ}$ & {$[0 \overline{1} 1]$} & & \\
\hline \multirow[t]{2}{*}{$\mathrm{Fi}$} & g & $\sim 2.0044$ & & & \multicolumn{3}{|c|}{ Not uniquely determined } & Bond-centered fluorine, $\mathrm{F}_{\mathrm{bc}}(+)$ & Present \\
\hline & $\mathbf{A}\left({ }^{19} \mathrm{~F}\right)$ & $\sim 4.8$ & $\sim 4.8$ & $\sim 8.2$ & $\sim 120^{\circ}$ & $\sim 145^{\circ}$ & $\perp[111]$ & & \\
\hline
\end{tabular}




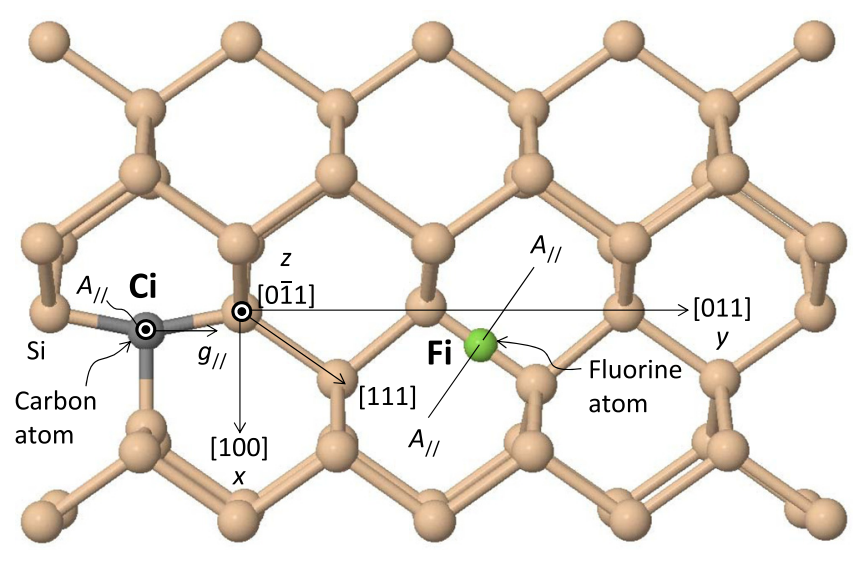

FIG. 4. Atomic models for the dry-etching damages of "Ci" and "Fi." "Ci" is a C-Si split interstitialcy or $\mathrm{C}_{i}(+)$, which is a variation of the G12 center observed in bulk $\mathrm{Si}^{10}$ " $\mathrm{Fi}$ " is a bond-centered fluorine or $\mathrm{F}_{\mathrm{bc}}(+)$, which was found in this study. The principal $Z(/ /)$ axes of the $g$ and A tensors of "Ci" and "Fi" are also shown.

the contrary, it was minimized in the $55^{\circ}$ direction for " $\mathrm{Fi}$ " [Fig. 3(b)], indicating that the symmetry axis of the ${ }^{19} \mathrm{~F}$ hyperfine splitting of " $\mathrm{Fi}$ " is perpendicular to the $\langle 111\rangle$ axis. This curious symmetry is reasonably explained by a bond-centered fluorine configuration. As shown in Fig. 4, a bond-centered fluorine forms a Si-F-Si bond along the $\langle 111\rangle$ axis. A non-bonding orbital of fluorine should be set to be perpendicular to the Si-F-Si bond, owing to the orthogonal relationship in the wave functions. Then, an unpaired electron in this orbital exhibited a $\langle 111\rangle$-normal axial symmetry for the ${ }^{19} \mathrm{~F}$ hyperfine splitting. The same relationship is also seen in the case of G12 and "Ci," where an unpaired electron occupied a non-bonding $\pi$-orbital perpendicular to a $s p^{2}$-bonding plane (see Fig. 4).

In Fig. 3(b), we simulated an angular map of "Fi." However, a lot of the simulated lines was "missing" in the experimental data. Such phenomenon was known in bulk crystals which were intentionally strained by 10 to $90 \mathrm{MPa} .^{14}$ The strain causes a preferential orientation of defects and the selection of observable ESR lines. ${ }^{10,11,14}$ Since there is a stronger strain (up to $600 \mathrm{MPa}$ ) inside Si MOSFETs, ${ }^{12}$ it may be reasonable to observe the "missing lines" in our EDMR centers. The occurrence of the "missing lines" was also reported in other MOSFETs ${ }^{8,15}$ and in interface systems ( $\mathrm{Si}-\mathrm{SiO}_{2}$ systems). ${ }^{16}$ In the latter case, an interface lowered the symmetry of the system, and in consequence, some orientations of a defect were prohibited. ${ }^{16}$ This situation seems plausible because "Fi" may be located near the $\mathrm{Si}_{-} \mathrm{SiO}_{2}$ interface. We note that in Figs. 3(b) and 3(c), the "missing lines" may be also observed in angular maps of "Ci" and other centers.

Due to the "missing lines," we could not uniquely determine the angular-map of "Fi." However, a number of our angular-map simulations based on the least-square fitting algorithm always converged to a similar result where the $\mathrm{A}\left({ }^{19} \mathrm{~F}\right)$ tensor was given as in Table I. The $A_{\|}$axis, which represents the direction of a $2 p$-orbital of fluorine, pointed approximately to the center of another $\mathrm{Si}-\mathrm{Si}$ bond, as shown in Fig. 4.

Another strong support for the model of a bond-centered fluorine is based on a theoretical consideration. For an isolated fluorine in $\mathrm{Si}, a b$ initio calculations ${ }^{6}$ predicted that a bond-centered fluorine is the most stable configuration when it is positively charged, while a tetrahedral interstitial configuration is the most favorable in the negative charge state. Judging from the fact that the coexisting defect of "Ci" is in the positive charge state, the same charge state should be expected for "Fi." Accordingly, the bond-centered fluorine is the most probable from a theoretical view point.

Furthermore, positive charge states of "Ci" and "Fi" are consistent with the distribution of carbon and fluorine. According to the SIMS results, these impurities should be distributed in the sub-surface region. Since our EDMR monitored the junction leakage currents from the depletion region [a white area in Fig. 2(a)], the location of the observed "Ci" and "Fi" centers is most likely within the depletion layer near or beneath the gate area, which is the edge of $p$-type region.

From the ${ }^{19} \mathrm{~F}$ hyperfine constants $\left(A_{/ /} \approx 8.2 \mathrm{mT}\right.$, $A_{\perp} \approx 4.8 \mathrm{mT}$, Table I) and the linear-combination-of-atomic-orbital analysis, ${ }^{13}$ we estimated $2 \%-3 \%$ of the wave-function distribution on a bond-centered fluorine atom. This situation is quite similar to the case of a bond-centered hydrogen atom (the AA9 center). ${ }^{17}$ In the AA9 center, a hyperfine splitting of ${ }^{1} \mathrm{H}$ revealed only $1.6 \%$ of the wave-function distribution on ${ }^{1} \mathrm{H}$. Instead, its wave function rather extended over the two Si neighbors by $21 \% \times 2$, causing a noticeable ${ }^{29}$ Si hyperfine signature of AA9. ${ }^{17}$ Although we could not resolve the ${ }^{29} \mathrm{Si}$ hyperfine splitting of "Fi," the similar distribution would be expected for a Si-F-Si bond.

In addition to the major defects "Ci" and "Fi", one can still find non-fitted experimental data in Figs. 3(b) and 3(c), which suggests the presence of other minor defects in our MOSFETs. We supposed that they are formed from carbon, fluorine, oxygen, and hydrogen, which are possible impurities introduced through the LSI processes. They have minor contributions to the junction leakage current, judging from their small signal intensities.

First, we point out that an angular map between "Ci" and G11 seemed to account for the F1 center $\left(\mathrm{F}_{n} V_{5}\right){ }^{13}$ The formation of $\mathrm{F} 1$ is quite reasonable because it is a final type of $\mathrm{F}_{n} V_{m}$ defects after annealing. ${ }^{13}$ The $\mathrm{F}_{n} V_{m}$ defects prevent the diffusion of fluorine atoms and lead to a sharper depth profile of fluorine. ${ }^{1,6,13}$ The SIMS profile in Fig. 1(b) shows such a behavior.

Second, angular maps observed in the higher-magneticfield side (337.5-339.0 mT) or low g factors $(g=1.994-2.003)$ might be fitted to hydrogen-related defects such as AA9 [Fig. 3(b)] and AA10 [Fig. 3(c)]. Both centers were found in proton-implanted $\mathrm{Si}$ substrates. ${ }^{17}$ The AA10 center was observed only in Czochralski-grown silicon, and it may be a complex of oxygen, carbon, and hydrogen impurities. ${ }^{17}$ However, in bulk crystals, they were only stable below $200 \mathrm{~K}$. Thus, we tentatively assume the enhancement of their thermal stability in Si MOSFETs. In fact, we observed the enhanced stability of "Ci" ( $\approx \mathrm{G} 12)$ in our devices, even though they vanished in $300-350 \mathrm{~K}$ isochronal annealing when they were in bulk samples. $^{10,11}$

In summary, using EDMR, we revealed the presence of carbon- and fluorine-interstitial defects (C-Si split interstitialcy and bond-centered fluorine) in practical $\mathrm{Si}$ MOSFETs. They were formed in the source/drain area and the edge of the gate area by fluorocarbon RIE and 
subsequent annealing processes, and they caused the junction leakage currents of the MOSFETs. We also found other types of minor dry-etching damages of carbon, fluorine, and hydrogen impurities, such as G11 (dicarbon interstitial), F1 $\left(\mathrm{F}_{n} V_{5}\right.$ cluster), and possibly AA9 (hydrogen interstitial) or AA10 (hydrogen-related defects). Surprisingly, they survived inside MOSFETs even after high-temperature thermal processes exceeding their annihilation temperatures in bulk samples. Our EDMR observations suggest an environmental difference between the insides of bulk silicon and LSIs.

${ }^{1}$ M. N. Kham, I. Matko, B. Chenevier, and P. Ashburn, J. Appl. Phys. 102, 113718 (2007).

${ }^{2}$ G. Roll, S. Jakschik, M. Goldbach, T. Mikolajick, and L. Frey, in Proceedings of the European Solid-State Device Research Conference (IEEE, 2010), p. 329.

${ }^{3}$ H. Komeda, M. Sato, A. Ishihama, K. Sakiyama, and T. Ohmi, in Proceedings of the 3rd International Symposium on Plasma ProcessInduced Damage (IEEE, 1998), p. 88.

${ }^{4}$ M. Wang and M. J. Kushner, J. Vac. Sci. Technol., A 29, 051306 (2011).

${ }^{5}$ F. Zirkelbach, B. Stritzker, K. Nordlund, J. K. N. Lindner, W. G. Schmidt, and E. Rauls, Phys. Rev. B 84, 064126 (2011).
${ }^{6}$ M. Diebel and S. T. Dunham, Phys. Rev. Lett. 93, 245901 (2004).

${ }^{7}$ J. Lee, C. Cho, J. Lee, S. Shin, J. Lee, D. Kwak, K. Lee, B. Roh, T. Chung, and K. Kim, in Proceedings of 32th European Solid-State Device Research Conference (IEEE, 2002), p. 571.

${ }^{8}$ T. Umeda, Y. Mochizuki, K. Okonogi, and K. Hamada, J. Appl. Phys. 94, 7105 (2003).

${ }^{9}$ Since the reduced RIE time was shorter than the period necessary for the complete etching, we must be careful of an incompleteness of the etching, when we adopt "process B."

${ }^{10}$ G. D. Watkins and K. L. Brower, Phys. Rev. Lett. 36, 1329 (1976).

${ }^{11}$ L. W. Song, X. D. Zhan, B. W. Benson, and G. D. Watkins, Phys. Rev. B 42, 5765 (1990).

${ }^{12}$ S. Ito, H. Namba, K. Yamaguchi, T. Hirata, K. Ando, S. Koyama, S. Kuroki, N. Ikezawa, T. Suzuki, T. Saitoh, and T. Horiuchi, Tech. Dig. of the Int. Electron Devices Meeting (IEEE, 2000), p. 247.

${ }^{13}$ T. Umeda, J. Isoya, T. Ohshima, S. Onoda, N. Morishita, K. Okonogi, and S. Shiratake, Appl. Phys. Lett. 97, 041911 (2010).

${ }^{14} \mathrm{M}$. Stalova, Identification of Defects in Semiconductors, Semiconductors and Semimetals Vol. 51B, edited by R. K. Willardson and E. R. Weber (Academic Press, 1998).

${ }^{15}$ M. S. Brandt, R. T. Neuberger, and M. Stutzmann, Appl. Phys. Lett. 76, 1467 (2000).

${ }^{16}$ A. Stesmans, B. Nouwen, and V. V. Afanas'ev, Phys. Rev. B 58, 15801 (1998).

${ }^{17}$ Yu. V. Gorelkinski and N. N. Nevinnyi, Physica B 170, 155 (1991). 